\title{
Aceitação e preferência de frutos para oviposição em duas populações de Ceratitis capitata (Diptera, Tephritidae)
}

\author{
Iara Sordi Joachim-Bravo ${ }^{1} \&$ Alberto Moreira da Silva-Neto ${ }^{1,2}$
}

\author{
1. Depto de Biologia Geral, Instituto de Biologia, Universidade Federal da Bahia (UFBA), Rua Barão do Geremoabo s/n, Campus \\ Universitário de Ondina, 40170-290, Salvador, BA. (ibravo@ufba.br) \\ 2. Bolsista PIBIC-CNPq.
}

\begin{abstract}
Acceptance and preference of fruits for oviposition in two Ceratitis capitata (Diptera, Tephritidae) populations. The influence of four host fruits, orange (Citrus sinensis L.), papaya (Carica papaya L.), mango (Mangifera indica L.) and apple (Malus domestica Borkh) on oviposition behavior of Ceratitis capitata (Wiedemann, 1824) was evaluated. Experiments were carried out on two C. capitata laboratory-reared populations: one with artificial diet for 10 years with periodic introduction of wild flies and other reared with artificial diet for 20 years without wild flies introduction. In acceptance experiments two pieces of a single fruit type were exposed to a group of 10 females; after 48 hours, these were changed by two new pieces of the same fruit type, and, in the end of the fourth day, the experiments were concluded. In preference experiments, two pieces of different fruits were offered to the females. These experiments were driven under the same conditions of the acceptance ones. Acceptance and preference experiments for both populations showed the following choice hierarchy: papaya > mango > orange > apple. The acceptance experiments showed no differences in the number of eggs/female/day laid in the first 48 hours and in the last 48 hours of the experiments. The amount of eggs in the two pieces of fruits offered to the females was similar. In spite of the two populations present similar behavior in relation to host hierarchy, the number of laid eggs was different, being larger for the population reared without wild flies introduction.
\end{abstract}

KEYWORDS. Fruitflies, oviposition, hierarchy, preference.

\section{INTRODUÇÃO}

Uma das relações mais estudadas entre os insetos fitófagos holometábolos e suas plantas hospedeiras é o comportamento de oviposição. A escolha do hospedeiro adequado por parte da fêmea no momento da postura é fundamental para a sobrevivência e sucesso da sua prole, pois as larvas possuem pouca mobilidade e dependem dos recursos nutritivos selecionados pelas fêmeas adultas no momento da oviposição (SINGER, 1986; Renwick, 1989). As fêmeas reconhecem os frutos hospedeiros por meio de sinais físicos e químicos destes. Podem utilizar estímulos visuais como cor, forma e tamanho e estímulos químicos, tais como nutrientes, substâncias voláteis das plantas, fagoinibidores e fagoestimulantes na busca e discriminação do hospedeiro (EISEMANN \& Rice, 1985; McInNis, 1989; Oi \& MAU, 1989; Messina, 1990). Em insetos polífagos, apesar da possibilidade de oviposição em vários hospedeiros diferentes, é comum as fêmeas exibirem uma hierarquia de preferência, sendo que alguns hospedeiros são sempre preferidos em relação a outros (THOMPSON, 1988).

A mosca Ceratitis capitata (Wiedemann, 1824) é uma praga agrícola de importância mundial (MALAVAsi $e t$ al., 1980). E polífaga, sendo registrada como praga de mais de 250 espécies de frutos e vegetais, multivoltínea e sem diapausa (Christenson \& Foote, 1960). No Brasil, infesta especialmente frutos introduzidos (MALAVAsi $e t$ al., 1980), e tem distribuição nas regiões Sudeste, Sul e Nordeste (MARTins et al., 1993).

Dados da literatura mostram que a criação das moscas-das-frutas por muitas gerações em laboratório causa mudanças no comportamento destas quando comparadas com populações selvagens. Geralmente, populações criadas em laboratório apresentam aumento na taxa reprodutiva, seus indivíduos atingem a maturidade mais rapidamente e possuem uma habilidade reduzida para o vôo (LEPPLA et al., 1983). Em relação ao comportamento alimentar e de oviposição, dados mostram uma menor capacidade de discriminação entre dietas artificiais e hospedeiros naturais (JoACHIM-BRAVO \& ZuCOLOTO, 1998). Entre outros cuidados, a freqüente introdução de moscas selvagens nas colônias de moscas de laboratório tem sido uma prática utilizada na intenção de manter a variabilidade genética e diminuir os efeitos deletérios causados pela endogamia. A relação inseto-planta pode contribuir para um melhor conhecimento sobre critérios de aceitação, preferência e inclusão de plantas hospedeiras, auxiliando no controle e manejo de tais pragas.

O objetivo foi comparar duas populações de $C$. capitata quanto ao comportamento de oviposição em relação a diferentes frutos hospedeiros. Uma das populações foi criada com dieta artificial por 10 anos, com a periódica introdução de indivíduos selvagens, e a outra mantida endogamicamente com dieta artificial por 20 anos.

\section{MATERIAL E MÉTODOS}

Estudou-se duas populações de $C$. capitata criadas em laboratório, uma aqui denominada população 1 é originária de moscas provenientes de uma criação mantida na Embrapa-Mandioca e Fruticultura (Cruz-das-Almas, Bahia) há aproximadamente 10 anos, com a introdução freqüente de indivíduos selvagens, provenientes de 
amêndoas infestadas (Terminalia catappa L., Combutaceae). A outra população (população 2) é originária de uma criação mantida no Laboratório de Nutrição de Insetos (FFCL/USP-Ribeirão-Preto) há aproximadamente 20 anos, sem introdução de indivíduos selvagens.

A manutenção das duas populações foi realizada no Laboratório de Ecologia Nutricional de Insetos (Instituto de Biologia,UFBA), de acordo com a metodologia descrita por Zucoloto (1987): adultos foram mantidos em gaiolas de armação de metal (20x20x25 cm) revestidas com tecido voal, contendo uma abertura lateral para introdução dos indivíduos e manuseio. Diariamente, colocava-se água e solução de sacarose e a cada dois dias introduzia-se uma dieta artificial contendo $6,5 \mathrm{~g}$ de lêvedo de cerveja, $11 \mathrm{~g}$ de açúcar, $2 \mathrm{~g}$ de ágar-ágar, $1 \mathrm{~g}$ de ácido cítrico, $1 \mathrm{ml}$ de nipagin e $100 \mathrm{ml}$ de água destilada (Zucoloto et al., 1979). As gaiolas foram mantidas em temperatura variável de $25-30^{\circ} \mathrm{C}$, recebendo iluminação durante 10 horas diárias para estimular a oviposição das fêmeas. Estas colocavam os ovos diretamente no tecido poroso que revestia a gaiola, posicionada sobre uma bandeja com água, onde estes foram coletados e colocados em placas de Petri com a mesma dieta oferecida aos adultos. As larvas foram mantidas em estufa, na ausência de luz, com temperatura aproximada de $25^{\circ} \mathrm{C}$ e umidade relativa de $70-80 \%$, conseguida com a utilização de quatro placas de Petri com água. No terceiro instar larval, as placas foram colocadas em caixas de acrílico $(11 \times 11 \times 3 \mathrm{~cm})$ contendo areia esterilizada, na qual permaneceram até o empupamento e posterior emergência dos adultos.

Os frutos testados nos experimentos quanto à aceitação e preferência foram: laranja (Citrus sinensis L.), mamão (Carica papaya L.), manga (Mangifera indica L.) e maçã (Malus domestica Borkh), que foram adquiridos em estado amadurecido. Para a verificação da aceitação dos frutos para oviposição em cada população, dez fêmeas e oito machos recém-emergidos foram colocados em uma caixa de acrílico $(11 \times 11 \times 3 \mathrm{~cm})$ e alimentados com água e dieta artificial ad libitum. No início do período de pico de oviposição das fêmeas (oitavo dia após a emergência para a população 2 e décimo quinto dia para a população 1$)$, dois pedaços $(5,0 \mathrm{~g})$ de um mesmo tipo de fruto foram introduzidos na caixa. A polpa do fruto foi coberta com papel alumínio de tal maneira que apenas a sua casca ficava exposta; esta foi perfurada dez vezes com alfinete, para que a dureza desta não interferisse na oviposição das fêmeas. Os pedaços tinham a mesma área de superfície em exposição e eram posicionados, na caixa, eqüidistantes entre si. As caixas foram iluminadas com lâmpadas fluorescentes, com fotoperíodo de 10 horas diárias. Após 48 horas, os frutos foram trocados por novos pedaços e no fim do quarto dia finalizou-se o experimento. Os pedaços de frutos foram identificados e congelados para posterior contagem de ovos. Para cada fruto testado foram feitas 10 repetições por população e os dados foram analisados com o uso de testes estatísticos não paramétricos.

A aceitação dos diferentes frutos para a oviposição das fêmeas foi estimada pelo número de ovos, sendo estes comparados pelo teste de Kruskal-Wallis, seguido pelo teste de comparações múltiplas de Dunn (HoLlandER \& Wolf, 1973). O teste de Wilcoxon (SiEgel, 1956) foi utilizado para verificar se havia diferenças no número de ovos entre os pedaços de um mesmo tipo de fruto. $\mathrm{O}$ teste de Mann-Whitney (SIEGEL, 1956) foi utilizado para verificar se a quantidade de ovos postos em um mesmo tipo de fruto nas primeiras 48 horas era similar ou não à quantidade de ovos postos nas 48 horas seguintes. Os testes foram feitos com o auxílio do programa GraphPad Sofware, instat guide to choosing and interpreting statistical tests, version, 1997, San Diego e para todos foi considerada a probabilidade crítica de $5 \%$ de erro.

Para a verificação da preferência para oviposição seguiu-se o mesmo procedimento realizado nos experimentos de aceitação. Foram oferecidos às fêmeas pedaços de dois tipos de frutos diferentes. Verificou-se a preferência de oviposição das fêmeas, quando expostas às seguintes combinações de frutos: mamão x laranja; mamão x maçã; mamão x manga; manga x laranja; manga $x$ maçã e laranja x maçã. Para análise dos dados, utilizou-se o teste estatístico de Wilcoxon a $5 \%$ de significância (SIEGEL, 1956). Foram feitas dez repetições para cada combinação de frutos em cada população.

\section{RESULTADOS}

Houve diferenças significativas no número de ovos encontrados nos diferentes frutos (Kruskal-Wallis, $\mathrm{p}<0,001)$. A análise pelo teste de comparações múltiplas de Dunn mostrou que em ambas as populações as fêmeas ovipositaram mais ovos no mamão e na manga em relação à laranja e maçã (fig. 1). Comparando-se mamão e manga,
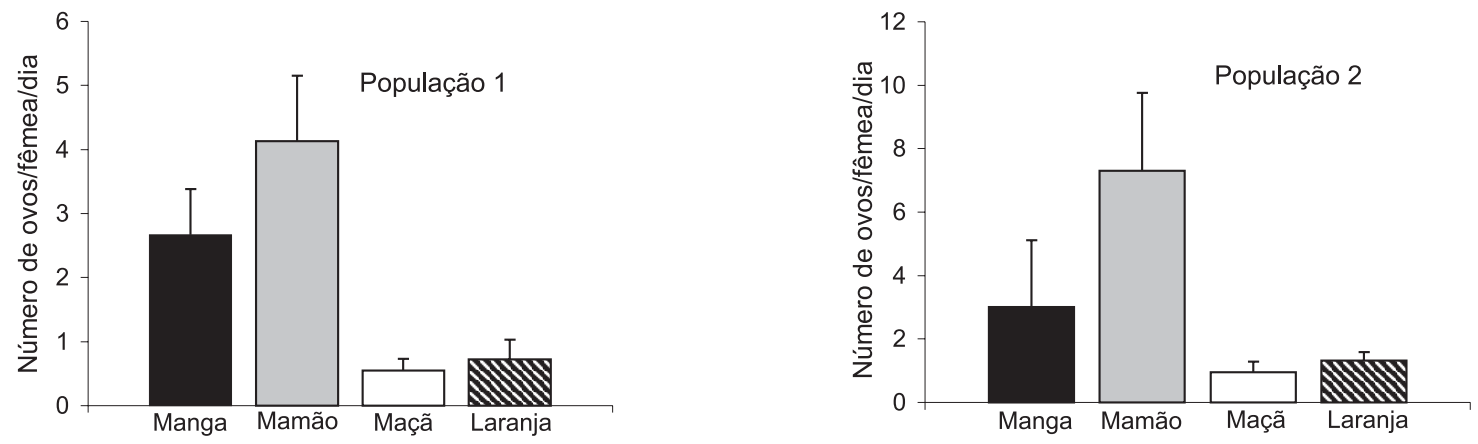

Fig. 1. Aceitação de frutos para oviposição das fêmeas de Ceratitis capitata expostas a pedaços de um só tipo de fruto. Os resultados representam as médias e desvios padrões de 10 repetições para cada população. Houve diferença estatística entre o número de ovos presentes nos diferentes frutos, obtendo-se as seguintes hierarquias de aceitação: população 1, mamão > manga > laranja = maçã; população 2, mamão > manga > laranja > maçã (Kruskal-Wallis, p< 0,05). 
Tabela I. Experimentos de aceitação da população 1 de Ceratitis capitata: número de ovos colocados em cada pedaço de fruto exposto às fêmeas durante 48 horas. Médias seguidas de letras distintas, em linha, dentro de cada intervalo de 48 horas, diferem estatisticamente entre si. Os resultados representam as médias e os desvios padrões de 10 repetições para cada fruto testado (Wilcoxon, p< 0,05 ).

\begin{tabular}{|c|c|c|c|c|c|c|}
\hline \multirow[b]{2}{*}{ Frutos } & \multicolumn{3}{|c|}{$\begin{array}{l}\text { Primeiras } 48 \text { horas } \\
\text { ovos/fêmea/dia }\end{array}$} & \multicolumn{3}{|c|}{$\begin{array}{c}\text { Últimas } 48 \text { horas } \\
\text { ovos/fêmea/dia }\end{array}$} \\
\hline & $1^{\circ}$ pedaço & $2^{\circ}$ pedaço & & $1^{\circ}$ pedaço & $2^{\circ}$ pedaço & \\
\hline Manga & $1,76 \pm 0,79^{\mathrm{a}}$ & $1,16 \pm 0,41^{\mathrm{a}}$ & $\mathrm{p}=0,070$ & $1,42 \pm 0,54^{\mathrm{a}}$ & $1,01 \pm 0,32^{\mathrm{a}}$ & $\mathrm{p}=0,051$ \\
\hline Mamão & $2,73 \pm 0,73^{\mathrm{a}}$ & $1,74 \pm 0,83^{b}$ & $\mathrm{p}=0,004$ & $2,09 \pm 0,74^{\mathrm{a}}$ & $1,55 \pm 0,75^{\mathrm{a}}$ & $\mathrm{p}>0,050$ \\
\hline Maçã & $0,35 \pm 0,23^{\mathrm{a}}$ & $0,27 \pm 0,21^{\mathrm{a}}$ & $\mathrm{p}=0,510$ & $0,27 \pm 0,18^{\mathrm{a}}$ & $0,21 \pm 0,12^{\mathrm{a}}$ & $\mathrm{p}=0,330$ \\
\hline Laranja & $0,38 \pm 0,27^{\mathrm{a}}$ & $0,30 \pm 0,16^{\mathrm{a}}$ & $\mathrm{p}=0,520$ & $0,40 \pm 0,32^{\mathrm{a}}$ & $0,23 \pm 0,19^{\mathrm{a}}$ & $\mathrm{p}=0,160$ \\
\hline
\end{tabular}

Tabela II. Experimentos de aceitação da população 2 de Ceratitis capitata: número de ovos colocados em cada pedaço de fruto exposto às fêmeas durante 48 horas. Médias seguidas de letras distintas, em linha, dentro de cada intervalo de 48 horas, diferem estatisticamente entre si. Os resultados representam as médias e os desvios padrões de 10 repetições para cada fruto testado (Wilcoxon, p< 0,05 ).

\begin{tabular}{|c|c|c|c|c|c|c|}
\hline \multirow[b]{2}{*}{ Frutos } & \multicolumn{3}{|c|}{$\begin{array}{c}\text { Primeiras } 48 \text { horas } \\
\text { ovos/fêmea/dia }\end{array}$} & \multicolumn{3}{|c|}{$\begin{array}{c}\text { Últimas } 48 \text { horas } \\
\text { ovos/fêmea/dia }\end{array}$} \\
\hline & $1^{\circ}$ pedaço & $2^{\circ}$ pedaço & & $1^{\circ}$ pedaço & $2^{\circ}$ pedaço & \\
\hline Manga & $1,91 \pm 1,52^{\mathrm{a}}$ & $0,72 \pm 0,51^{\mathrm{b}}$ & $\mathrm{p}=0,042$ & $2,26 \pm 2,20^{\mathrm{a}}$ & $1,14 \pm 1,31^{\mathrm{a}}$ & $\mathrm{p}=0,105$ \\
\hline Mamão & $3,64 \pm 1,39^{\mathrm{a}}$ & $3,66 \pm 2,39^{a}$ & $\mathrm{p}=0,980$ & $3,22 \pm 1,54^{\mathrm{a}}$ & $4,42 \pm 1,96^{\mathrm{a}}$ & $\mathrm{p}>0,290$ \\
\hline Maçã & $0,57 \pm 0,36^{\mathrm{a}}$ & $0,46 \pm 0,27^{\mathrm{a}}$ & $\mathrm{p}=0,330$ & $0,38 \pm 0,26^{\mathrm{a}}$ & $0,54 \pm 0,17^{\mathrm{a}}$ & $\mathrm{p}=0,160$ \\
\hline Laranja & $0,74 \pm 0,30^{\mathrm{a}}$ & $0,56 \pm 0,17^{\mathrm{a}}$ & $\mathrm{p}=0,180$ & $0,67 \pm 0,34^{\mathrm{a}}$ & $0,68 \pm 0,36^{\mathrm{a}}$ & $\mathrm{p}=0,625$ \\
\hline
\end{tabular}

Tabela III. Experimentos de aceitação das populações 1 e 2 de Ceratitis capitata: comparação do número de ovos colocados nas primeiras e nas últimas 48 horas de ambas as populações. Os resultados são as médias e desvios padrões de 10 repetições para cada fruto testado. Não houve diferença estatística entre os dados comparados em nenhuma das duas populações (Mann-Whitney a 5\% de significância).

\begin{tabular}{|c|c|c|c|c|c|c|}
\hline \multirow[b]{2}{*}{ Frutos } & \multicolumn{3}{|c|}{ População 1} & \multicolumn{3}{|c|}{ População 2} \\
\hline & $\begin{array}{l}\text { Primeiras } 48 \text { horas } \\
\text { ovos/fêmea/dia }\end{array}$ & $\begin{array}{l}\text { Últimas } 48 \mathrm{l} \\
\text { ovos/fêmea/c }\end{array}$ & & $\begin{array}{l}\text { Primeiras } 48 \text { horas } \\
\text { ovos/fêmea/dia }\end{array}$ & $\begin{array}{l}\text { Últimas } 48 \text { horas } \\
\text { ovos/fêmea/dia }\end{array}$ & \\
\hline Manga & $2,92 \pm 0,83$ & $2,42 \pm 0,68$ & $\mathrm{p}=0,190$ & $2,63 \pm 1,61$ & $3,40 \pm 2,80$ & $\mathrm{p}=0,578$ \\
\hline Mamão & $4,62 \pm 1,19$ & $3,64 \pm 1,20$ & $\mathrm{p}=0,075$ & $7,29 \pm 2,95$ & $7,34 \pm 2,42$ & $\mathrm{p}=0,853$ \\
\hline Maçã & $0,62 \pm 0,26$ & $0,48 \pm 0,22$ & $\mathrm{p}=0,217$ & $1,03 \pm 0,52$ & $0,91 \pm 0,29$ & $\mathrm{p}=0,481$ \\
\hline Laranja & $0,76 \pm 0,32$ & $0,68 \pm 0,42$ & $\mathrm{p}=0,545$ & $1,30 \pm 0,31$ & $1,35 \pm 0,54$ & $\mathrm{p}=0,795$ \\
\hline
\end{tabular}

houve maior postura no primeiro $(\mathrm{p}<0,05)$. Entre laranja e maçã, houve diferença na aceitação das fêmeas das diferentes populações. As fêmeas da população 1 não exibiram diferença no número de ovos ovipositados em ambos os frutos ( $p>0,05)$; já as da população 2 ovipositaram maior quantidade de ovos na laranja em relação à maçã $(\mathrm{p}<0,05)$. Verificou-se que, na maioria das comparações realizadas, uma quantidade de ovos similar foi ovipositada nos dois pedaços de um mesmo tipo de fruto oferecidos às fêmeas (tabs. I, II), tanto na população de laboratório como na selvagem. A comparação do número de ovos colocados nas primeiras e nas últimas 48 horas de ambas as populações (tab. III), mostrou que as fêmeas não alteraram a quantidade de ovos postos no decorrer do experimento.

Os resultados referentes aos experimentos de preferência de oviposição (fig. 2) mostraram que em ambas as populações as fêmeas preferiram ovipositar no mamão em relação às outras três frutas testadas, tanto nas primeiras 48 horas (população 1: mamão $\mathrm{x}$ manga, $p=0,0020$; mamão $x$ maçã, $p=0,0020$; mamão $x$ laranja, $\mathrm{p}=0,0020$; população 2: mamão x manga, $\mathrm{p}=0,0195$; mamão x maçã, $\mathrm{p}=0,0020$; mamão x laranja, $\mathrm{p}=0,0020$, teste de Wilcoxon), como nas últimas 48 horas (população 1: mamão x manga, $\mathrm{p}=0,0195$; mamão $\mathrm{x}$ maçã, $\mathrm{p}=0,0020$; mamão $\mathrm{x}$ laranja, $\mathrm{p}=0,0020$; população 2: mamão $\mathrm{x}$ manga, $\mathrm{p}=0,0020$; mamão $\mathrm{x}$ maçã, $\mathrm{p}=0,0020$; mamão $\mathrm{x}$ laranja, $\mathrm{p}=0,0020$, teste de Wilcoxon). A manga foi a segunda fruta quanto ao número de ovos ovipositados, recebendo número maior de ovos do que a maçã e a laranja nos testes de escolha, tanto nas primeiras 48 horas (população 1: manga $x$ maçã, $p=0,0020$; manga $x$ laranja, $p=0,0020$; população 2: manga $\mathrm{x}$ maçã, $\mathrm{p}=0,0020$; manga $\mathrm{x}$ laranja, $\mathrm{p}=0,0020$, teste de Wilcoxon), como nas últimas 48 horas (população 1: manga x maçã, $p=0,0020$; manga $x$ laranja, $\mathrm{p}=0,0020$; população 2: manga $\mathrm{x}$ maçã, $\mathrm{p}=0,0020 ;$ manga $\mathrm{x}$ laranja, $\mathrm{p}=0,0020$, teste de Wilcoxon). Nas comparações entre maçã e laranja houve sempre preferência para a laranja em ambas as populações, nas primeiras 48 horas (população 1: laranja x maçã, $\mathrm{p}=0,0098$; população 2: laranja x maçã, $\mathrm{p}=0,0371$, teste de Wilcoxon) e nas últimas 48 horas (população 1: laranja x maçã, $\mathrm{p}=0,0020$; população 2: laranja x maçã, $p=0,0039$, teste de Wilcoxon). De acordo com os resultados obtidos pode-se estabelecer a seguinte hierarquia de preferência: mamão>manga $>$ laranja>maçã. 

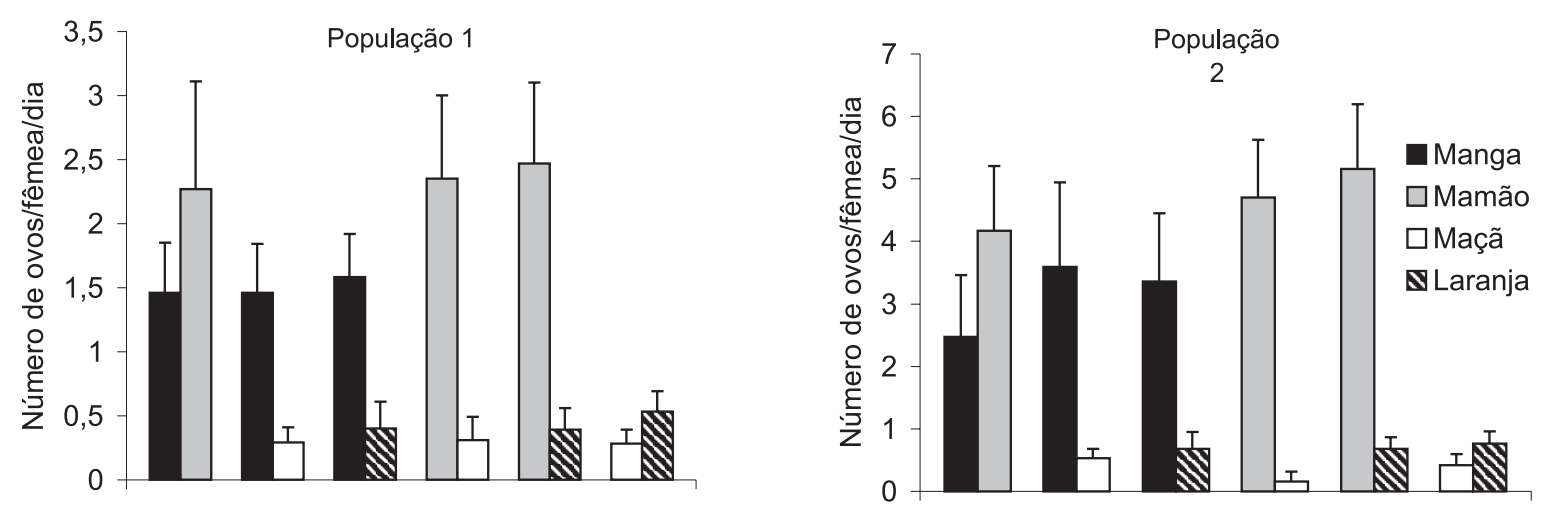

Fig. 2. Preferência de frutos para oviposição das fêmeas de Ceratitis capitata expostas a quatro tipos de frutos em combinações de dois a dois. Os resultados mostram as médias e desvios padrões de 10 repetições para cada população. Houve diferença estatística para cada combinação em ambas as populações (Wilcoxon, p<0,05).

\section{DISCUSSÃO}

As fêmeas de $C$. capitata apresentaram hierarquia de preferência em relação aos hospedeiros testados. Evidenciou-se também que ambas as populações estudadas apresentaram hierarquia de preferência similar. Esses dados corroboram os de outro estudo que testou a existência de hierarquia na fase inicial do período de oviposição de fêmeas individuais de C. capitata (JoACHIM-BRAvo et al., 2001a).

A existência de hierarquia de preferência por hospedeiros em insetos fitófagos polífagos tem sido evidenciada também em outros insetos (THOMPSON, 1988). Em C. capitata parece haver um componente genético envolvendo a preferência por frutos hospedeiros, uma vez que, tanto neste estudo, que envolveu experimentos com fêmeas em grupo, quanto em outro realizado com fêmeas individuais (JoACHIMBRAVo et al., 2001a), foi evidenciada a mesma hierarquia de preferência em duas populações distintas. Em ambos os casos, uma das populações usadas era proveniente de uma criação mantida em laboratório, sem contato com frutos e sem a introdução de indivíduos selvagens há mais de 20 anos. Isso sugere que a adaptação às condições de laboratório parece não ter afetado a capacidade das fêmeas dessa população de reconhecer frutos e estabelecer preferência para oviposição. Essa hierarquia de preferência pode estar relacionada à qualidade nutricional do fruto para larvas de $C$. capitata. Os frutos mamão e manga permitiram melhor desenvolvimento das larvas em relação a maçã e laranja (JoACHIM-Bravo \& Zucoloto, 1997; JoAChim-Bravo et al., 2001a, b).

A existência de correlação entre preferência de oviposição e performance larval para alguns insetos é relatada por THOMPSON (1988). MAYHEW (2001) relatou um novo tipo de correlação entre preferência de oviposição e melhor alimentação para adultos e não para as larvas. Tais relatos contraditórios tornam a questão polêmica, uma vez que a diversidade de hábitos alimentares dos insetos holometábolos e a diversidade de parâmetros analisados dificultam a observação de padrões gerais. SADEGHI \& GILBERT (1999) registraram a existência de uma variabilidade individual, em relação à existência de correlação entre preferência e performance, dentro de uma população e YAMAGA \& OHGUSHi (1999) constataram que, em alguns casos, ocorre uma variação anual na correlação positiva preferência-performance no campo. Parece haver predominância dessa correlação em insetos especialistas em comparação a insetos polífagos (Barros \& Zucoloto, 1999; Craig et al., 2000), pois estes últimos têm uma menor capacidade de discriminação de hospedeiros. Isso seria uma vantagem em ambientes imprevisíveis e facilitaria a mudança e a adaptação a novos hospedeiros (Fletcher \& Prokopy, 1991). Apesar de ser polífaga, C. capitata mostrou ter capacidade de discriminar hospedeiros, evidenciando uma hierarquia de preferência. Se considerarmos que a preferência da fêmea baseia-se na escolha de recursos adequados à sobrevivência da prole, pode-se supor vantagens adaptativas deste comportamento, visto que poderia escolher os de melhor qualidade.

A similaridade na hierarquia de preferência de oviposição de populações diferentes, uma exclusivamente composta por indivíduos mantidos em laboratório e outra mista (composta por indivíduos de laboratório + selvagens), denota semelhança comportamental entre as fêmeas das duas populações. Pode-se inferir que, para estudos de comportamento de oviposição como os aqui realizados, a utilização de populações compostas exclusivamente de moscas criadas em laboratório não é inviável.

A avaliação da distribuição dos ovos nos diferentes pedaços de um mesmo tipo de fruto mostrou que houve uma distribuição eqüitativa destes. Este fato corrobora o trabalho de PAPAs et al. (1990) que mostraram que a fêmea é capaz de perceber frutos nos quais já houve uma oviposição prévia e colocam menos ovos nestes em relação a outros nos quais ainda não 
houve postura. Tal comportamento é atribuído ao feromônio de marcação depositado pelas fêmeas no momento da oviposição (PAPAJ et al., 1990). Esses resultados dão suporte aos experimentos de preferência e demonstram que a distribuição de ovos nestes últimos não foi aleatória.

Evidenciou-se, também, uma similaridade na quantidade de ovos postos nas primeiras e nas últimas 48 horas dos experimentos de aceitação. Uma diminuição no número de ovos postos poderia ser observada com o avanço da idade das fêmeas. Provavelmente, o tempo de duração do experimento não permitiu essa constatação.

Comparando-se as duas populações, verificouse que as fêmeas da população 1 sempre ovipositaram menos que as da população 2 , independente da hierarquia de preferência. Esse dado corrobora os de Leppla et al. (1983) e JoACHIM-Bravo et al. (2001b), nos quais foram constatados que fêmeas de laboratório produzem e ovipositam em maior quantidade que as selvagens. Nesse caso, portanto, as fêmeas da população 1 estariam apresentando resultados similares às de populações selvagens, estudadas nos referidos trabalhos, em relação a um menor número de ovos produzidos quando comparadas às populações criadas há muitos anos em laboratório. Isso nos leva a sugerir que a maior capacidade de postura (ou maior produção de ovos) da população 2 seja uma característica genética selecionada nesta população que tem sido mantida isolada por aproximadamente 20 anos. A população 1, mantida com a introdução freqüente de indivíduos selvagens, provavelmente não sofreu seleção direcional para uma maior produtividade. Tais registros são relevantes, pois a utilização de insetos criados continuamente em laboratório para estudos de comportamento, fisiologia e ecologia tem sido motivo de questionamentos com relação à aplicabilidade dos resultados obtidos para as populações naturais. Sem dúvida a criação contínua em laboratório acarreta mudanças fisiológicas e comportamentais relevantes, como aumento na taxa reprodutiva, maturação mais rápida, redução da habilidade para o vôo, menor capacidade de discriminação entre dietas artificiais e hospedeiros naturais, aumento do tempo de desenvolvimento dos imaturos, aumento da competição larval e aumento da mortalidade dos adultos (LePPla et al., 1983; Ecomonopoulos, 1992; JoACHIM-Bravo \& Zucoloto, 1998), apesar de alguns comportamentos, como os descritos neste trabalho, não sofrerem influência da criação contínua e isolada. A utilização de criação mista, com adição de indivíduos selvagens, poderia garantir maior confiabilidade na generalização dos resultados de estudos fisiológicos e comportamentais.

Agradecimentos. Ao PIBIC/CNPq pela bolsa de iniciação científica concedida.

\section{REFERÊNCIAS BIBLIOGRÁFICAS}

Barros, H. C. H. \& Zucoloto, F. S. 1999. Performance and host preference of Ascia monuste (Lepidoptera, Pieridae). Journal of Insect Physiology, Oxford, 40:7-14.
Christenson, L. D. \& Foote, R. H. 1960. Biology of fruit flies. Annual Review of Entomology, Palo Alto, 5:171-192.

Craig, T. P.; ItAmi, J. K. et al. 2000. The influence of host plant variation and intraespecific competition on oviposition preference and offspring performance in the host races of Eurosta solidaginis. Ecological Entomology, London, 25:7-18.

Ecomonopoulos, A. P. 1992. Adaptation of the mediterranean fruit fly (Diptera: Tephritidae) to artificial rearing. Journal of Economic Entomology, Lanham, 85:753-758.

Eisemann, C. H. \& Rice, M. J. 1985. Oviposition behaviour of Dacus tryoni: the effects of some sugars and salts. Entomologia Experimentalis et Applicata, Dordrecht, 39:61-71.

Fletcher, B. S. \& Prokopy, R. J. 1991. Host location and oviposition in tephritid fruit flies. In: BAILEy, W. J. \& RidsDill-Smith, J. Reproductive behaviour of insects: individuals and populations. London, Chapman \& Hall. p. $139-171$.

Hollander, M. \& Wolf, D. A. 1973. Nonparametric statistical methods. New York, John Wiley \& Sons. p. $184-193$.

Joachim-Bravo, I. S.; Fernandes, O. A. et al. 2001a. Oviposition preference hierarchy in Ceratitis capitata (Diptera, Tephritidae): influence of female age and experience. Iheringia, Série Zool., Porto Alegre, (91):93-100.

$2001 \mathrm{~b}$. Oviposition behavior of Ceratitis capitata Wiedemann (Diptera: Tephritidae): association between oviposition preference and larval performance in individual females. Neotropical Entomology, Londrina, 30(4):559564.

Joachim-Bravo, I. S. \& Zucoloto, F. S. 1997. Oviposition preference and larval performance in Ceratitis capitata. Revista Brasileira de Zoologia, Curitiba, 14(4):795802 .

_-_ 1998. Performance and feeding behavior of Ceratitis capitata: comparison of a wild population and laboratory population. Entomologia Experimentalis et Applicata, Dordrecht, 87:67-72.

Leppla, N. C.; Huettel, M. D. et al. 1983. Strategies for colonization and maintenance of the Mediterranean fruit fly. Entomologia Experimentalis et Applicata, Dordrecht, 33:89-96.

Malavasi, A.; Morganti, J. S. \& Zucchi, R. A. 1980. Biologia de "moscas-das-frutas". I. Lista de hospedeiros e ocorrência. Revista Brasileira de Biologia, Rio de Janeiro, 40(1):9-16.

Martins, D. S.; Alves, F. L. \& Zucchi, R. A. 1993. Levantamento de moscas-das-frutas (Diptera: Tephritidae) na cultura do mamoeiro (Carica papaya L.) no norte do Estado do Espírito Santo. Anais da Sociedade Entomológica do Brasil, Londrina, 22(2):373-379.

MAYHEW, P. J. 2001. Herbivore host choice and optimal bad motherhood. Trends in Ecology \& Evolution, Amsterdam, 16(4):165-167.

McInNis, D. O. 1989. Artificial oviposition sphere for Mediterranean fruit flies (Diptera: Teprhitidae) in field cages. Journal of Economic Entomology, Lanham, 82(5): 1382-1385.

Messina, F. J. 1990. Components of host choice by two Rhagoletis species (Diptera: Tephritidae) in Utah. Journal of the Kansas Entomological Society, Lawrence, 63(1):80-87.

Oı, D. H. \& MaU, R. F. L. 1989. Relationship of fruit ripeness to infestation in "Sharwil" avocados by the Mediterranean fruit fly and oriental fruit fly (Diptera: Tephritidae). Journal of Economic Entomology, Lanham, 82(2):556-560.

Papaj, D. R.; Roitberg, B. D. et al. 1990. Effect of marking pheromone on clutch size in Mediterranean fruit fly. Physiological Entomology, Oxford, 15:463-468.

Renwick, J. A. A. 1989. Chemical ecology of oviposition in phytophagous insects. Experientia, Barel, 45:223-228.

Sadeghi, H. \& Gilbert, F. 1999. Individual variation in oviposition preference, and its interaction with larval performance in a insect predator. Oecologia, Heidelberg, 118: $405-411$. 
Siegel, S. 1956. Nonparametric statistics for the behavioral sciences. New York, MacGraw-Hill. 340p. Singer, M. C. 1986. The definition and measurement of oviposition preference in plant-feeding insects. In: Miller, J. R. \& Miller, T. A. eds. Insect-plant interactions. New York, Springer-Verlag. p.66-94.

Thоmpson, J. N. 1988. Evolutionary ecology of the relationship between oviposition preference and performance of offspring in phytophagous insects. Entomologia Experimentalis et Applicata, Dordrecht, 47:3-14.
YAmaga，Y. \& Ohgushi, T. 1999. Preference-performance linkage in a herbivorous lady beetle: consequences of variability of natural enemies. Oecologia, Heidelberg, 119:183-190.

Zucoloto, F. S. 1987. Feeding habits of Ceratitis capitata: can larvae recognize a nutritional effective diet? Journal of Insect Physiology, Oxford, 33:349-353.

Zucoloto, F. S.; Puschel, S. \& Message, C. M. 1979. Valor nutritivo de algumas dietas artificiais para Anastrepha obliqua (Diptera: Tephritidae). Boletim de Zoologia, São Paulo, 4:75-80. 